\title{
Marfan syndrome: improved clinical history results in expanded natural history
}

\author{
Reed E. Pyeritz, MD, PhD ${ }^{1}{ }^{1}$
}

Life expectancy for a person with Marfan syndrome has essentially doubled over the past four decades. During this period, the clinical histories of the organs managed routinely have improved, and will continue to be. Prominent examples are the eyes, the heart and aorta, and some features of the skeletal system. Meanwhile, the natural histories of organ systems that have not been subjected to treatment need to be described. This is particularly important as due to the improved life span many symptoms and organ systems are only recently being recognized as being intrinsic to Marfan syndrome. Examples are the distal aorta and peripheral arteries, ventricular function, the central nervous system, sleep apnea, and adiposity. As a result, each person with Marfan syndrome will need to be evaluated and followed by more specialists than previously.
Moreover, the coordinator of diagnostic testing and clinical referral must be aware of the expanded phenotype as people with Marfan syndrome age and the importance of life-long management of classical and novel features. The benefits of increased longevity and its consequences need to be addressed by investigators, health-care providers, and patients alike.

Genetics in Medicine (2019) 21:1683-1690; https://doi.org/10.1038/s41436018-0399-4

Keywords: Marfan syndrome; natural history; clinical history; pleiotropy

\section{INTRODUCTION}

"Disease is very old, and nothing about it has changed. It is we who change as we learn to recognize what was formerly imperceptible."

-Jean-Martin Charcot (1825-1893), French neurologist

During the first half of the twentieth century, the fundamental clinical features of the Marfan syndrome (MFS; OMIM 154700) were described: dislocation of the ocular lens, arachnodactyly, abnormal histopathology of the aortic wall, dilatation of the first portion of the aorta associated with aortic regurgitation, and dissection of the ascending aorta. The latter two complications led to early death. In 1972, when there was no effective treatment for any aspect of MFS, life expectancy was reduced by $30-40 \%$ in males and females, with a mean age of death of 32 years, with wide variation. ${ }^{1}$

Not until the description and application of composite graft surgery of the aortic root in the mid to late 1970s did life expectancy begin to increase. ${ }^{2}$ Any insights about the phenotype of MFS until that time would be termed natural history. Since then, the evolution of aspects of phenotype that have been subject to therapies, for example, the risk of aortic dissection after composite graft surgery, is termed clinical history. The clinical histories through middle age of various features of MFS are increasingly well documented. ${ }^{3}$

This review addresses current natural history, particularly of the many new features of MFS that have emerged since life expectancy has begun to approach that of the general population. The result has been both a marked expansion of what we now understand the MFS phenotype to be, and the need to consider the natural history of these previously unrecognized clinical features. The older literature (pre-2000) is replete with case reports and case series that claim to be only about MFS, but often include conditions that are now known to be separate, such as Loeys-Dietz syndrome. As a result, this discussion will favor more recent reports involving patients with confirmed MFS.

\section{Diagnosis of the Marfan syndrome}

The criteria used to label someone as affected with the MFS have been subject to three international conferences over a quarter century. ${ }^{4}$ The most recent incorporates the discovery that variants in FBN1, which encodes the microfibrillar protein fibrillin-1, are the cause of MFS. Several features are important in their own right (ectopia lentis, involvement of the aortic root), while a criterion that subsumes ten other 
features is given equal weight with the others. The natural histories of some of these features are well understood, and since a number are subject to therapies, their clinical histories have also been scrutinized. One effect is that diagnostic criteria change with age, and so the diagnosis in a child may be more difficult than in an adolescent or adult. The presence of a pathogenic variant in FBN1 or the certain diagnosis of MFS in a relative makes establishing a diagnosis straightforward regardless of the age of the patient.

\section{The consequences of longer survival in MFS}

Several fundamental issues inform the discussions. First, how do the features of MFS present in the typical patient change over time? For example, do parts of the aorta distal to the ascending portion develop dilatation or dissection and at what frequencies? Second, what "new" features emerge as patients survive into the fifth decade and beyond? For example, how frequent and severe is sleep apnea and how is it best managed? Third, how are issues common in any aging individual affected by MFS? For example, many people develop degenerative arthritis as they age, but is this more common, of earlier onset, and more severe in people with MFS? Finally, to what extent are studies in the general population relevant to MFS? For example, are risk factors for atherosclerosis more or less relevant? Not all of the important issues will have definitive answers. This review recommends areas in need of further research.

\section{Organ systems}

\section{The eye}

Ectopia lentis is one of the findings used as a fundamental diagnostic criterion. Yet, it is present in only about one-half of people otherwise diagnosed with MFS. ${ }^{5}$ Once thought to be congenital, ectopia lentis can develop during both childhood and adulthood. ${ }^{6}$ The lens tends to migrate superiorly and the zonules tend to stretch but remain intact. Removal of the dislocated lens and correcting visual acuity without a lens requires long-term follow-up. ${ }^{7}$ Implantation of an intraocular lens is increasingly being considered once the ectopic lens is removed. Medium-term results seem encouraging. ${ }^{8}$

Myopia worse than -3 diopters is quite common at early age, due to the extended length of the globe, although this is modulated somewhat by a flattened cornea. Whether myopia worsens with age, or if other refraction abnormalities change more often or worsen with age than in the general population is unclear. Because the lens is unusually flat to begin with in MFS, Lasik for improvement of visual acuity should not be considered. ${ }^{9}$

A variety of other ocular problems develop with time. For example, cataracts are common and tend to develop at earlier ages than in the general population. ${ }^{10}$ Management has typically been by standard approaches, to satisfactory results. An increased risk of retinal detachment occurs at any age, likely due to the increased axial length of the globe. ${ }^{11}$ This is an issue requiring counseling throughout life, and protective eyewear is essential.

\section{Cardiovascular system}

No part of the anatomy generates as much concern in MFSand academic investigation-as the heart and vasculature. The main stimulus, of course, is the major role disease of the ascending aorta has played in premature death. Neither variant type nor family history is a satisfactory predictor of the severity of cardiovascular involvement, in part because of the exceptional intrafamilial variability. Aggressive management-medications to reduce stress on the ascending aorta or impact the underlying pathophysiology directly, routine imaging, and prophylactic aortic root replacement-are routine for any MFS patient diagnosed before a catastrophic event occurs. ${ }^{12}$ With these aggressive treatments for the aorta, attention is required on the long-term consequences on the heart, the rest of the arterial system, and even the venous system.

Aorta. Both $\beta$-adrenergic and angiotensin receptor blockade reduce the rate of aortic root dilatation in many young people with MFS. ${ }^{13,14}$ Adding losartan to adequate $\beta$-adrenergic blockade results in no enhancement of protection. ${ }^{15}$ Even without treatment, the stiffness of the aorta increases with age somewhat more in MFS than in matched healthy controls. Chronic $\beta$-adrenergic blocker treatment reduces the stiffness index in young patients whereas an angiotensin receptor blocker did not. ${ }^{16}$ Higher stiffness indices resulted in more adverse clinical outcomes. Elastic properties of the ascending aorta are abnormal beginning in childhood. Additional factors may interact to enhance aortic root dilatation.

One area that has markedly evolved and benefited from medium-term longitudinal analyses is cardiothoracic surgery of the dilated sinuses of Valsalva. Elective versus emergency surgery on the ascending aorta is clearly preferable. ${ }^{17}$ Cardiothoracic surgery on the ascending aorta has evolved from composite graft repair pioneered by Hugh Bentall to an aortic valve-sparing approach pioneered by Tirone David. One advantage of preserving the native aortic valve over a mechanical one is the lack of a need for anticoagulation. But how will the spared aortic valve in this currently preferred approach to surgery on the aortic root hold up over decades? ${ }^{18}$ Some suggest a nontrivial rate of reoperation. ${ }^{19}$ What is the impact of replacing either just the proximal ascending aorta or the entire ascending aorta into the arch with an inelastic Dacron tube on the rest of the thoracic aorta and its major branches? ${ }^{20}$ There has been some research on pulse wave velocity and distal aortic stiffness, but no long-term studies. ${ }^{21}$ Some people have increased tortuosity of the vertebral, splenic, or renal arteries, and over time will this predispose to aneurysm formation or dissection? ${ }^{22}$ Two studies have examined the risk of peripheral arterial aneurysms. ${ }^{23,24}$ Both concluded that occurrences were frequent and in need of screening and, if present, regular monitoring. Peripheral aneurysms were more frequent in patients who had previous repair of the aortic root. In a relatively small sample, half of detected peripheral aneurysms eventually required intervention, ${ }^{23}$ raising concerns for all aging patients with MFS. 
Both the natural and clinical histories of aortic dissection require better definition. Acute aortic dissection in MFS occurs more frequently in the winter and spring and during daylight hours, just as in the general population. ${ }^{25}$ Over a short term of 3.6 years, among 423 subjects with MFS in the GenTAC registry, $1.6 \%$ suffered an aortic dissection. ${ }^{26}$ The risk of dissection of the aorta distal to the arch (type B) remains to be defined accurately, particularly over decades. Among MFS subjects in GenTAC, one-third developed a type $\mathrm{B}$ dissection first. ${ }^{26}$ Is there an increased risk of a type $\mathrm{B}$ dissection after repair of the ascending aorta? What is the best approach to managing a chronic dissection of the descending thoracic aorta? Determining at what diameter to repair the dilated, dissected descending aorta remains ill defined. Many centers do not favor endovascular treatment for type B dissections in the MFS. ${ }^{27}$ Will older people with MFS have a heightened risk of developing abdominal aortic aneurysms? Acute dissection affecting the descending thoracic and abdominal aortas can produce ischemia to visceral and pelvic organs due to vascular compromise. Ischemia to the spinal cord can compromise neurologic and muscular function. Chronic dissection can produce the same adverse effects, with their occurrence often dependent on progressive dilatation of the dissected aortic segments. There is a need for both improved medical and surgical therapy of the dissected aorta. $\beta$-adrenergic blockade remains the primary medical therapy.

Mitral valve. Other common cardiac manifestations-prolapse of the mitral and tricuspid valves in particular-require long-term clinical follow-up to understand their natural histories. Mitral valve prolapse is an important diagnostic criterion, ${ }^{4}$ is often present in childhood, and can progress to be of serious medical concern. Factors that contribute to progression, its relationship to aortic dilatation or genotype, and if echocardiography is the best means for following it are all uncertain. For moderate or severe mitral regurgitation, mitral valve replacement was standard decades ago, but mechanical valves require chronic anticoagulation. Gradually, annuloplasty and leaflet repair were introduced with encouraging results. ${ }^{28}$ Now, mitral valve repair is effective initially and over the midterm, ${ }^{29}$ but further studies of clinical history are needed.

Pulmonary artery. The main pulmonary artery can be dilated in about $10 \%$ of children with MFS and is associated with other severe cardiovascular involvement. ${ }^{30}$ However, long-term complications have not been studied, but are likely infrequent.

Ventricular function. In the past, left ventricular failure was typically due to chronic aortic, or occasionally mitral, regurgitation. Now that surgery can reduce valvular issues, it has become clear that some ventricles deteriorate on their own with either systolic or diastolic dysfunction. ${ }^{31}$ Understanding the predictors of ventricular failure, such as genotype, is essential to better management. Is there a relationship between skeletal muscle and cardiac muscle weakness? What medications might be effective in preventing primary cardiac muscle deterioration? What are the effects of $\beta$-adrenergic blockade, angiotensin receptor blockade, or other drugs?

Electrophysiology. Electrophysiologic complications can occur. In one study of 80 adults with MFS that included ambulatory electrocardiogram (ECG) monitoring, half had some ventricular arrhythmia at baseline, and $8 \%$ had a ventricular tachycardia event on follow-up, including several sudden cardiac deaths. ${ }^{32}$ Issues in need of research are the incidence of events, the predisposing factors (such a type of variant, valvular pathology, cardiomyopathy), how patients should be monitored, does physical exertion promote dysrhythmia, and whether prophylactic medication should be considered.

Physical exertion and exercise. Whether exertion should be limited, and to what extent, has been discussed extensively but with little data to justify conclusions. Acute type A dissections in males under 50 years of age were associated with physical exercise in two-thirds in whom a trigger could be determined, but less than $1 \%$ of the study population had MFS. ${ }^{33}$ In $16 \%$ of males, but no females, dissection occurred during sexual intercourse. Some young people become competitive athletes before the diagnosis of MFS is made, and several have suffered lethal aortic dissection during exertion. Anyone with MFS should be counseled against competition or exertion at near maximal capacity, and activities that risk collisions. Similarly, static (isometric) exercise increases blood pressure more than dynamic (isokinetic) exercise and should also be discouraged. However, these recommendations are based on no systematically obtained data. The threshold between what level of exertion is acceptable to maintain satisfactory cardiopulmonary and muscular condition, and what contributes to more rapid aortic root expansion or dissection is unknown, and in any event will vary among individuals. ${ }^{34}$ Benefits of mild to moderate exercise include physical conditioning, improved bone density, and lowering of blood pressure at rest. However, potentially deleterious effects of exercise include aortic wall remodeling, endothelial dysfunction, and vascular smooth muscle function, which may contribute to the threat of dissection and variations in risk among patients. $^{35}$

Pregnancy. The cardiovascular risks of pregnancy for a woman with MFS have been examined over the past few decades. Most studies could document individual cases of aortic dissection during the late stages of gestation and, particularly, in the 6 months postpartum. ${ }^{36-38}$ Both type A and type B dissections occurred, so basing prepregnancy counseling solely on the diameter of the aortic root is imprecise. Nonetheless, recommending against pregnancy when the root diameter is greater than $40 \mathrm{~mm}$ is common and based on convincing data. ${ }^{36,39}$ Now that valve-sparing aortic root repair obviates the need for warfarin, pregnancy after prophylactic 
aortic root repair must be studied. Because angiotensin receptor blockade is contraindicated during pregnancy, treatment with $\beta$-adrenergic blockade should be prescribed. Preliminary, retrospective evidence suggests that for women with equivalent aortic root diameters, those who complete one or more pregnancies experience a more rapid rate of aortic root dilatation. ${ }^{40}$

Preliminary data suggest that two factors predispose to postpartum dissection: the use of angiotensin converting enzyme inhibitors and breast feeding. Breast feeding stimulates persistent elevation of oxytocin, which is deleterious to the aortic wall. This is obviously a difficult issue for those women who would prefer to breast feed. The preferred method of delivery is also subject to debate. For women with only a mildly dilated aortic root, vaginal delivery if otherwise viewed as safe should be undertaken. The long-term consequences of pregnancy on the health of the mother, especially whether she has a heightened risk of cardiovascular complications, are uncertain. ${ }^{40}$

Infants of women with MFS are more likely to be preterm and small for gestational age and to be delivered by Cesarean section. $^{41}$

\section{Skeletal system}

Variants in FBN1 cause a remarkably wide range of skeletal findings. Indeed, some variants cause several forms of short stature, including geleophysic dysplasia. Other variants (e.g., R2726W) cause only the skeletal features typical of MFS, but without any other findings. ${ }^{42}$ Perhaps the first recognizable phenotypic features are tall stature and arachnodactyly. ${ }^{43}$ However, other well-recognized abnormalities in children can progress with age and lead to secondary problems. Joint laxity can lead to instability and subluxation. Physical therapy can be a life-long necessity. Pain when walking due to pes planus and calcaneoplano valgus typically does not diminish with age. Hammer toes and bunions are common with increasing age. Complications of joint laxity are instability, tendon and ligament tears, decreased physical activity, and eventually degenerative arthritis. ${ }^{44}$ In adults with MFS, virtually any joint can cause discomfort and limit range of motion. ${ }^{45}$ For example, the temporomandibular joint is frequently lax, leading to subluxation and pain.

Protrusio acetabulae, in which the hip socket protrudes into the pelvic cavity, is common enough in MFS to be one of the diagnostic skeletal criteria. Specific radiologic measurements are used to diagnose and follow this congenital deformity. The prevalence is $15-27 \%$ depending on the measurement used, and increases until bone maturity. ${ }^{46}$ Symptomatic treatment in children is potentially effective, whereas surgical management in adults, including arthroplasty, often is not. ${ }^{47}$

In the general population, vertebral column deformity typically ceases to progress once the skeleton matures. In MFS, however, both scoliosis and abnormal kyphosis can become accentuated with age, leading to pain, limitation of movements, neurologic compromise, and restrictive pulmonary disease. A key goal is to prevent spinal column deformity from progressing during childhood and adolescence, through regular monitoring once a curve reaches 20 degrees, and bracing as the curve progresses. An aim is to prevent a scoliotic curve from rapidly progressing. Management needs to be more aggressive than in idiopathic scoliosis, involving magnetic growth rods in children with an eventual need for spinal fusion. ${ }^{48}$ One complication of scoliosis is leg length discrepancy, which is often unrecognized and a cause of gait disturbance and hip pain. Thoracic kyphosis can be exaggerated and, in my experience, may respond to bracing for scoliosis.

Pectus excavatum can reduce pulmonary capacity and, when severe, interfere with venous return and cardiac output. Repairing pectus excavatum too early in childhood risks having the deformity reoccur as the ribs continue to elongate. ${ }^{49}$ The experience with pectus excavatum repair in adults with MFS, such as by the Nuss procedure, is limited. ${ }^{50}$

Bone mineral status has been analyzed only in crosssectional studies, with markedly different conclusions, i.e., no differences with age-matched controls, osteopenia at the femoral neck in males but not females, bone mineral density decreased in both adult men and women, and no differences for adults of either gender. ${ }^{51}$ Evaluations of children have been limited to date, so natural history is unknown, although one study suggested reduced bone mineral density. ${ }^{52}$ Whether patients are predisposed to fractures, and how they respond to standard therapies, need to be studied. To what extent osteopenia progresses to osteoporosis and at what ages is unclear. In the absence of fractures, patients likely do not need routine screening or medical treatments.

Joint hypermobility may contribute to precocious osteoarthritis, but requires longitudinal study with a focus on preventive management.

Excessively tall stature is typically more of a psychosocial concern than a medical one, particularly in girls. Hormonal treatment in early adolescence has been utilized for decades to promote early epiphyseal closure. ${ }^{53,54}$ While adult height can be reduced, long-term effects on other skeletal characteristics or perceived quality of life have not been evaluated.

\section{Respiratory system}

The first complication in MFS, even before the diagnosis is established, may be a spontaneous pneumothorax. Eventually, about $5-10 \%$ of patients will experience this problem, which is due to rupture of a bleb in a lung, typically in the apex. ${ }^{55}$ Predisposing factors include trauma to the chest and rapid changes in atmospheric pressure. Small leaks may reseal if the trapped air is evacuated, but leaks that do not reseal require resection of the ruptured bleb and pleurodesis. Activities that may predispose to pneumothorax include flying in unpressurized aircraft and scuba diving. Cystic changes, of which blebs are an overt manifestation, are common and may only be evident on histopathology. ${ }^{56}$ The extent to which patients develop symptoms of obstructive lung disease remains uncertain. 
Anterior chest development can involve pectus carinatum, excavatum, or a combination of the two. The former typically reduces lung volume. Spinal thoracic deformity, both scoliosis and lordosis, also can reduce lung volume. Both repair of pectus and of severe vertebral deformities may be required to prevent further decrease in pulmonary volume. In people with MFS without significant thoracic deformity, lung volumes, with a normal range based on height, are interpreted as reduced. However, height is exaggerated by leg length, thus if the normal range of lung volume is based on sitting height, volumes in adults with MFS are frequently within an expected range. ${ }^{57}$

\section{Sleep}

About 25\% of the American adult population suffers from acute insomnia. Many individuals transition to chronic insomnia, with frequency increasing with age, and a major contributing factor is sleep disordered breathing. In MFS, obstructive and central sleep apnea are common and, just as in the general population, are more common in males and increase with age. ${ }^{58,59}$ In MFS, several factors are important. Cardiovascular abnormalities, including aortic dilatation, decreased left ventricular ejection fraction, and atrial fibrillation, correlate with presence and severity of sleep apnea. ${ }^{60}$ Obstructive sleep apnea in particular is enhanced by craniofacial abnormalities and increased upper airway collapsibility. ${ }^{61}$ The importance of obesity is unclear, but in my experience contributes to apnea. All adults with MFS should have a sleep study and appropriate interventions, such as continuous positive airway pressure at night. Correction of some of the contributing craniofacial factors, such as retrognathia and malocclusion, should be considered in childhood. ${ }^{62,63}$

\section{Abdominal organs}

No primary malfunctions of the liver, gastrointestinal tract, pancreas, spleen (or entire immunologic system), or kidneys are known, either as a baseline or as people with MFS age. The only secondary complications in any of these organs result from decreased blood flow due to a type B aortic dissection.

Simple cysts in both the liver and kidney are relatively common and occasionally can enlarge quite substantially. ${ }^{64}$ While there is no recorded impact on organ function, the presence of cysts is important to understand so that radiologists and other physicians (as well as patients) do not become unduly concerned.

\section{Urogenital organs}

Gynecologists observe that women with MFS are more likely to develop uterine prolapse, especially associated with childbirth. Bladder incontinence may occur earlier and more severely. In both women and men, voiding difficulties can be related to dural ectasia. None of these issues have been studied in detail.

\section{Nervous system}

The principal manifestation of MFS affecting the nervous system is dural ectasia, which is stretching of the dura under apparently normal pressures of the cerebrospinal fluid. ${ }^{65}$ Anterior meningoceles often protrude through dilated neural foramina. Rather than narrowing caudally as in the typical spinal column, in MFS the dimensions increase. There have also been instances of enlarged dural spaces over the cerebral cortex. Dural ectasia is included as an important criterion for diagnosis. ${ }^{4}$

The natural history of dural ectasia has been studied in only a few subjects. A consistent and reliable method for quantifying the anatomic deformities is necessary. Dural ectasia is often evident in childhood. ${ }^{66}$ Still unknown is the rate of progression, both of the anatomic features and symptoms, ${ }^{67}$ although in a small series of adults (mean age 49.6 years), there was no progression over 10 years. ${ }^{68}$ The principal symptoms are radicular pain, focused on whichever lumbosacral nerve roots are involved. Both acute and chronic postural cerebral hypotension occur, the former associated with sudden leakage of cerebrospinal fluid and the severity of the latter likely associated with the size of the lumbosacral meningoceles, and hence the space for cerebrospinal fluid to pool when one is upright. ${ }^{69}$

Administering epidural anesthesia can be complicated by lumbosacral dural ectasia. ${ }^{70}$ Whether this becomes more of a problem as older people with MFS require more surgical procedures is not clear.

For dural ectasia, there are neither medical nor surgical therapies that are effective over either the short or long term. This is an area in need of aggressive investigation.

The brain itself seems unaffected, but no formal study exists to separate the common effects of aging from those seen in MFS, either in type or age of onset. Cerebral circulation can be characterized by unusual tortuosity of vertebral and carotid arteries, but these are not typically associated with neurologic features. ${ }^{22}$ Whether there is an increased risk of cerebral vessel dissection or dilatation with age is unclear.

\section{Skeletal muscular system}

Skeletal muscles have long been thought to be primarily affected in MFS, mainly because of the obvious paucity of muscle bulk. Moreover, the muscle mass inversely correlates with the overall severity of the phenotype in any given patient. Adults report more weakness and fatigue than age-matched people, although the effects of medication and recommended reduction of physical activity can contribute to the differences. A study of bioenergetics showed that MFS patients required a higher energy consumption to achieve the same work output as controls. ${ }^{71}$ This finding needs to be replicated and the results followed over time. The few reports of muscle biopsies show signs of myopathy in muscles not impacted by neurogenic abnormalities associated with dural ectasia.

\section{Integument}

Congenital and acquired hernias are clearly more common in MFS than the general population. Diaphragmatic hernias of various types are evident at birth or become problematic in infancy and childhood. Inguinal hernias tend to appear later. 
Incisional hernias are also a concern, so procedures performed laparascopically should prove advantageous.

\section{Adiposity}

Although it has been inadequately documented, the asthenic habitus of childhood and adolescence often transitions to one of central obesity. Increased body mass index seems not markedly different from the general population, but more patients need to be followed. ${ }^{72}$ In MFS, the majority of the central deposition of adipose tissue is not subcutaneous, but visceral in the abdomen. Visceral adiposity (i.e., white fat) surrounds the major organs and leads to the classic protuberant abdomen. Subcutaneous and visceral fat are distinct in terms of their impact on appearance and metabolism, and risks for complications. Importantly, visceral fat predisposes to insulin resistance and type 2 diabetes mellitus, and in MFS is associated with aortic complications. ${ }^{72}$

In both mice and cell culture, fibrillin-1 affects differentiation of adipocytes and an increase in fat tissue. However, in humans the causes of a shift to an increase in adipose tissue and its preferential deposition in the abdomen are not clear. Additionally, the impact that these transitions, now that longevity is increasing, will have on morbidity and mortality is also unclear. Because aggressive physical exercise is not indicated, other efforts in addition to moderate exercise, such as persistent calorie restriction, should be pursued.

\section{Reproduction}

As with any hereditary condition, genetic counseling is essential. Now that people of both sexes are surviving to reproductive age, they need to understand the chances of having an affected child and any alternatives to standard child-bearing. In recent years their options have increased beyond avoiding pregnancy completely or utilizing prenatal diagnosis and selective termination. In vitro fertilization and preimplantation genetic diagnosis are routinely available. However, concerns of couples, particularly women with MFS, have increased as well. Whether having genetic counseling or not impacts reproductive decisions deserves further investigation. Preliminary studies suggest that having a pregnancy may accelerate dilatation of the aortic root over subsequent years, and if confirmed, this fact must become a part of reproductive genetic counseling. ${ }^{36}$

\section{Psychological issues}

Concern about MFS and its many aspects and how to cope can express itself at one end of a spectrum as a focus and commitment to recommendations about management or to resignation and depression at the other. A person's position along this spectrum can vary over time. Unhappiness with restrictions on employment and exercise may worsen perspectives. When surveyed with standard psychosocial evaluative instruments, about half of adults expressed symptoms of depression unrelated to potential adverse effects of medication. Anxiety waxes and wanes, typically accentuated by pain and disability and major medical and surgical events, such as cardiothoracic surgery. ${ }^{73}$ Fears, whether appropriate or not, often begin during childhood and adolescence. Approaches to managing exaggerated fear and anxiety have not been studied in MFS. For example, do effective psychotherapeutic measures applied in adolescence help to alleviate fears later in life? The health professionals coordinating overall care for MFS and ones dealing with primary care, need to keep the emotional concerns in mind. All studies should be judged against "normal" fears in young and older people.

\section{Quality of life}

All physical and psychological considerations factor into a person's perception of quality of life. For example, chronic pain occurs in the majority of adults, limits activity, and has no definitive treatment. ${ }^{73,74}$ Additionally, psychological concerns negatively influence quality of life, particularly in older men with MFS. Executive function difficulties, such as mental fatigue, were more common in MFS. ${ }^{75}$ Other issues, termed psychosocial, attempt to explain how a person's actual and perceived condition impacts their interactions with people, institutions, and the environment. Studies have applied both quantitative and qualitative tools, but most have been limited by small sample size, poor response rate, or both. Furthermore, all studies have been cross-sectional and not longitudinal. Issues other than those directly related to the MFS provide a backdrop to health concerns. Greater than $80 \%$ of knowledgeable patients perceived MFS as negatively impacting their lives. Nearly all viewed MFS as a severe or potentially lethal condition. In the largest survey to date, 389 patients completed a survey that found numerous predictors of better quality of life: less severe MFS, no depression, being married, being a college graduate, having had fewer prior surgeries, and no more than moderate alcohol use. In multivariate analysis, the only two predictive factors were having health insurance and being employed. Meanwhile, health-related quality of life scores were significantly lower than general population norms. Understanding if perceptions of quality of life worsen over time if insurance or employment is lost, or if any other factors intervene, would be relevant.

Short-term or long-term involvement with support groups can be helpful, but has not been studied in MFS.

\section{Other issues with aging}

There are many other aspects of "normal" aging that have not been examined in MFS. Examples are atherosclerosis and neoplasia in its many forms. In this regard, anecdotal evidence carries little weight, and large-scale studies require the cooperation of many centers and health-care professionals.

\section{CONCLUSION}

All aspects of MFS will become clearer as the genetic and environmental factors are better understood, especially as knowledge of pathogenesis improves. Natural history continues to evolve as people age. The clinical history of each of the manifestations both emerges and evolves as new 
treatments are applied and also as people age. Both histories require careful research, preferably longitudinal. Centers that focus on MFS, committed investigators, funding agencies, and patient support groups, such as The Marfan Foundation (marfan.org) in the United States, can and should contribute to this research.

The general issues discussed here are relevant to many pleiotropic monogenic disorders especially those in which earlier diagnosis and effective treatments have led to increased life expectancy. For example, in hereditary hemorrhagic telangiectasia, progressive involvement of the gastrointestinal tract and liver become increasing concerns as people survive pulmonary and cerebral arteriovenous malformations.

\section{ACKNOWLEDGEMENTS}

The author recognizes the tremendous support of his patients and colleagues over the decades. Jane Tumpson provided invaluable editorial support. No grant funding supported the production of this article.

\section{DISCLOSURE}

R.E.P. declares no conflicts of interest.

\section{REFERENCES}

1. Murdoch JL, Walker BA, Halpern BL, et al. Life expectancy and causes of death in the Marfan syndrome. N Engl J Med. 1972;286:804-808.

2. Gray JR, Bridges $A B$, West RR, et al. Life expectancy in British Marfan syndrome populations. Clin Genet. 1998;54:124-128.

3. Pyeritz RE. Recent progress in understanding the natural and clinical histories of the Marfan syndrome. Trends Cardiovasc Med. 2016;26:423-428.

4. Loeys BL, Dietz HC, Braverman AC, et al. The revised Ghent nosology for the Marfan syndrome. J Med Genet. 2010;47:476-485.

5. Drolsum L, Rand-Hendriksen S, Paus B, et al. Ocular findings in 87 adults with Ghent-1 verified Marfan syndrome. Acta Ophthalmol. 2015;93:46-53.

6. Sandvik GF, Vanem TT, Rand-Hendriksen S, Cholidis S, Sæthre M, Drolsum L Ten-year reinvestigation of ocular manifestations in Marfan syndrome. Clin Exp Ophthalmol 2018; https://doi.org/10.1111/ ceo.13408. Accessed 18 December 2018.

7. Utz VM, Coussa RG, Traboulsi El. Surgical management of lens subluxation in Marfan syndrome. J AAPOS. 2014;18:140-146.

8. Luebke J, Reinhard T, Agostini H, Boeringer D, Eberwein P. Long-term follow-up after scleral lens fixation in patients with Marfan syndrome. BMC Ophthalmol. 2017;17:235-240.

9. Dietz H. Marfan syndrome. In: Adam MP, Ardinger HH, Pagon RA et al., (eds.) GeneReviews. Seattle, WA: University of Washington; 2017.

10. Konradsen TR, Zetterström C. A descriptive study of ocular characteristics in Marfan syndrome. Acta Ophthalmol. 2013;91:751-755.

11. Rahmani S, Lyon AT, Fawzi AA, et al. Retinal disease in Marfan syndrome: from the Eye Consortium of Chicago. Ophthalmic Surg Lasers Imaging Retina. 2015;46:936-941.

12. Hoskoppal A, Menon S, Trachtenberg F, et al. Predictors of rapid aortic root dilatation and referral for aortic surgery in Marfan syndrome. Pediatr Cardiol. 2018;39:1453-61.

13. Shores J, Berger KR, Murphy EA, Pyeritz RE. Chronic B-adrenergic blockade protects the aorta in the Marfan syndrome: a prospective, randomized trial of propranolol. N Engl J Med. 1994;330:1335-1341.

14. Lacro RV, Dietz HC, Sleeper LA, et al. Randomized trial of atenolol versus losartan in children and young adults with Marfan syndrome. N Engl J Med. 2014;371:2061-2071.

15. Muiño-Mosquera L, De Nobele $S$, Devos $D$, et al. Efficacy of losartan as add-on therapy to prevent aortic growth and ventricular dysfunction in patients with Marfan syndrome: a randomized, double-blind clinical trial. Acta Cardiol. 2017;72:616-624.
16. Tuerney ESS, Levine JC, Sleeper LA, et al. Influence of aortic stiffness on aortic-root growth rate and outcome in patients with the Marfan syndrome. Am J Cardiol. 2018;121:1094-1101.

17. Song $H K$, Kindem M, Bavaria JE, et al. Long-term implications of emergency versus elective proximal aortic surgery in patients with Marfan syndrome in the Genetically Triggered Thoracic Aortic Aneurysms and Cardiovascular Conditions Consortium Registry. J Thorac Cardiovasc Surg. 2012;143:282-286.

18. Volguina IV, Miller DC, LeMaire SA, et al. Valve-sparing and valvereplacing techniques for aortic root replacement in patients with Marfan syndrome: analysis of early outcome. I Thorac Cardiovasc Surg. 2009;137:1124-1132.

19. Attenhofer Jost $\mathrm{CH}$, Connolly HM, Scott CG, et al. Aortic root surgery in Marfan syndrome: medium-term outcome in a single-center experience. $J$ Heart Valve Dis. 2017;26:45-53.

20. Rylski B, Bavaria JE, Beyersdorf F, et al. Type A aortic dissection in Marfan syndrome: extent of initial surgery determines long-term outcome. Circulation. 2014;129:1381-1386.

21. Westenberg JJM, Scholte AJHA, Vaskova Z, van der Geest RJ, Groenink M, Labadie G, et al. Age-related and regional changes of aortic stiffness in the Marfan syndrome: assessment with velocity-encoded MRI. J Magn Reson Imaging. 2011;34:526-531.

22. Morris SA, Orbach DB, Geva T, et al. Increased vertebral artery tortuosity index is associated with adverse outcomes in children and young adults with connective tissue disorders. Circulation. 2011;124:388-396.

23. Yetman AT, Roosevelt GE, Veit N, Everitt MD. Distal aortic and peripheral arterial aneurysms in patients with Marfan syndrome. J Am Coll Cardiol. 2011;58:2545-2545.

24. Gaertner S, Alembik Y, Cordeanu E-M, et al. Should we systematically screen for peripheral arterial aneurysms in all patients with Marfan syndrome? Int J Cardiol. 2014;172:e94-5.

25. Siddiqi HK, Luminais SN, Montgomery D, et al. Chronobiology of acute aortic dissection in the Marfan syndrome (from the National Registry of Genetically Triggered Thoracic Aortic Aneurysms and Cardiovascular Conditions and the International Registry of Acute Aortic Dissection). Am J Cardiol. 2017;119:785-789.

26. Weinsaft JW, Devereux RB, Preiss LR, Feher A, Roman MJ, Basson CT, et al. Aortic dissection in patients with genetically mediated aneurysms: Incidence and predictors in the GenTAC registry. J Am Coll Cardiol. 2016;67:2744-2754.

27. Pacini $D$, Parolare $A$, Berretta $P$, et al. Endovascular treatment of type $B$ dissection in Marfan syndrome: is it worthwhile? Ann Thorac Surg. 2013:95:737-749

28. Gillinov AM, Hulyalkar A, Cameron DE, et al. Mitral valve operation in patients with the Marfan syndrome. J Thorac Cardiovasc Surg. 1994;107:724-731.

29. Bernhardt AM, Treede H, Detter $C$, et al. Results of modern mitral valve repair in patients with Marfan syndrome. Thorac Cardiovasc Surg. 2014;62:35-41.

30. Stark VC, Huemmer M, Olfe J, et al. The pulmonary artery in pediatric patients with Marfan syndrome: an underestimated aspect of the disease. Pediatr Cardiol 2018; https://doi.org/10.1007/s00246-018-1880-1.

31. Hetzer R, Siegel G, Walter EMD. Cardiomyopathy in Marfan syndrome. Eur J Cardio-Thorac Surg. 2016;49:561-568.

32. Aydin A, Adsay BA, Sheikhzadeh S, et al. Observational cohort study of ventricular arrhythmia in adults with Marfan syndrome caused by FBN1 mutations. PLoS ONE. 2013;8:e81281.

33. Gansera L, Deutsch O, Szameitat L, Eichinger W, Gansera B. Aortic dissections type $A$ during sexual intercourse in male patients: accident or systematic coincidence? Examination of 365 patients with acute aortic dissection within 20 years. Thorac Cardiovasc Surg. 2016;64:133-136.

34. Cheng A, Owens D. Marfan syndrome, inherited aortopathies and exercise. What is the right answer? Heart. 2015;101:752-757.

35. de Waard. Marfan on the move. J Am Heart Assoc. 2017; https://doi.org/ 10.1161/JAHA.117.007465. Accessed 18 December 2018.

36. Roman MJ, Pugh NL, Hendershot TP, et al. Aortic complications associated with pregnancy in Marfan syndrome: the NHLBI National Registry of Genetically Triggered Thoracic Aortic Aneurysms and Cardiovascular Conditions (GenTAC). J Am Heart Assoc. 2016; https:// doi.org/10.1161/JAHA.116.004052. Accessed 18 December 2018.

37. Pyeritz RE. Maternal and fetal complications of pregnancy in the Marfan syndrome. Am J Med. 1981;71:784-790.

38. Goldand S, Wlkayam U. Pregnancy and Marfan syndrome. Ann Cardiothorac Surg. 2017;6:642-653. 
39. Kuperstein R, Cahan T, Yoeli-Ullman R, Ben Zerky S, Shinfeld A, Simchen MJ. Risk of aortic dissection in pregnant patients with the Marfan syndrome. Am J Cardiol. 2017:119:132-137.

40. Renard M, Muiño-Mosquera L, Manalo EC, et al. Sex, pregnancy and aortic disease in Marfan syndrome. PLoS One. 2017;12:e018166.

41. Curry RA, Gelson E, Swan L, et al. Marfan syndrome and pregnancy: maternal and neonatal outcomes. BJOG. 2014;121:610-617.

42. Reyes-Hernández OD, Placios-Reyes C, Chávez-Ocaña S, et al. Skeletal manifestations of Marfan syndrome associated to heterozygous R2726W FBN1 variant: sibling case report and literature review. BMC Musculoskelet Disord. 2016;17:79.

43. Stheneur C, Tubach F, Joneaux M, et al. Study of phenotype evolution during childhood in Marfan syndrome to improve clinical recognition. Genet Med. 2014;16:246-250.

44. Al Kaissi A, Zwettler E, Granger R, et al. Musculo-skeletal abnormalities in patients with Marfan syndrome. Clin Med Insights Arthritis Musculoskelet Disord. 2013;6:1-9.

45. Grahame R, Pyeritz RE. Marfan syndrome: joint and skin manifestations are prevalent and correlated. Br J Rheumatol. 1995;34:126-131.

46. Sponseller PD, Jones KB, Ahn NU, et al. Protrusio acetabulae in Marfan syndrome: age-related prevalence and associated hip function. J Bone Joint Surg Am. 2006;88:486-495.

47. Thakkar SC, Foran JR, Meers SC, Sponseller PD. Protrusion acetabula and total hip arthroplasty in patients with Marfan syndrome. J Arthroplasty. 2012;27:776-782

48. Gjola JP, Sponseller PD, Shah SA. et al. Spinal deformity correction in Marfan syndrome versus adolescent idiopathic scoliosis. Spine (Phila Pa 1976). 2012;37:1558-1565.

49. Arn PH, Scherer LR, Haller JA Jr, Pyeritz RE. Outcome of pectus excavatum in patients with Marfan syndrome and in the general population. J Pediatr. 1989;115:954-958.

50. Kelly RE, Goretsky MJ, Obermeyer R, et al. Twenty-one years experience with minimally invasive repair of pectus excavatum by the Nuss procedure in 1215 patients. Ann Surg. 2010;252:1072-1081.

51. Giampetro PF, Peterson M, Schneider R, et al. Assessment of bone mineral density in adults and children with Marfan syndrome. Osteoporos Int. 2003;14:559-563.

52. Grover M, Brunetti-Pierri, Belmont J, Hennekam R, et al. Assessment of bone mineral status in children with Marfan syndrome. Am J Med Genet. 2012;158A:2221-2224.

53. Rochiccioli P, Tauber MT. Treatment of tall stature of Marfan disease with a delayed-action analog of somatostatin (SMS 201-995). J Genet Hum. 1988;36:255.

54. Rozendaal L, le Cessie S, Wit JM, et al. Growth-reductive therapy in children with Marfan syndrome. J Pediatr. 2005;147:674-679.

55. Hall JR, Pyeritz RE, Dudgeon DL, Haller JA Jr. Pneumothorax in the Marfan syndrome: prevalence and therapy. Ann Thorac Surg. 1984;37:500-504.

56. Dyhdalo K, Farver C. Pulmonary histologic changes in Marfan syndrome: a case series and literature review. Am J Clin Pathol. 2011;136:857-863.

57. Streeten EA, Murphy EA, Pyeritz RE. Pulmonary function in the Marfan syndrome. Chest. 1987;91:408-412.
58. Cistulli PA, Sullivan CE. Sleep-disordered breathing in Marfan's syndrome. Am Rev Respir Dis. 1993;147:645-648.

59. Rybczynski M, Koschyk D, Karmeier A, et al. Frequency of sleep apnea in adults with the Marfan syndrome. Am J Cardiol. 2010;105:1836-1841.

60. Kohler M, Blair E, Risby P, et al. The prevalence of obstructive sleep apnoea and its association with aortic dilatation in Marfan's syndrome. Thorax. 2009;64:162-166.

61. Cistulli PA, Gotsopooulos H, Sullivan CE. Relationship between craniofacial abnormalities and sleep-disordered breathing in Marfan's syndrome. Chest. 2001;120:1455-1460.

62. Taddei $M$, Alkhamis $N$, Tagariello $T$, et al. Effects of rapid maxillary expansion and mandibular advancement of upper airways in Marfan's syndrome children: a home sleep study and cephalometric evaluation. Sleep Breath. 2015;19:1213-1220.

63. Paolini V, Lomabardo EC, Placidi Fruvolo G, Cozza P, Laganà G. Obstructive sleep apnea in children with Marfan syndrome: relationships between three-dimensional palatal morphology and apnea-hypopnea index. Int J Pediatr Otorhinol. 2018;112:6-9.

64. Chow K, Pyeritz RE, Litt HI. Abdominal visceral findings in patients with Marfan syndrome. Genet Med. 2007;9:208-212.

65. Pyeritz RE, Fishman EK, Bernhardt BA, Siegelman SS. Dural ectasia is a common feature of the Marfan syndrome. Am J Hum Genet. 1988:43:726-732.

66. Knirsch W, Kurtz C, Häffner N, et al. Dural ectasia in children with Marfan syndrome: a prospective, multicenter, patient-control study. Am J Med Genet. 2006;140A:775-781.

67. Foran JRH, Pyeritz RE, Dietz HC, Sponseller PD. Characterization of the symptoms associated with dural ectasia in the Marfan patient. Am J Med Genet. 2005;134A:58-65.

68. Mesfin A, Ahn NU, Carrino JA, Sponseller PD. Ten-year clinical and imaging follow-up of dural ectasia in adults with Marfan syndrome. Spine J. 2013;13:62-67.

69. Ferrante E, Citterio A, Savino A, Santalucia P. Postural headache in a patient with Marfan's syndrome. Cephalalgia. 2003;23:552-555.

70. Vacula BB, Gray C, Hofkamp MP, et al. Epidural analgesia complicated by dural ectasia in the Marfan syndrome. Proc (Bayl Univ Med Cent). 2016;29:385-386

71. Crilley JG, Bendahan D, Boehm EA. Investigation of muscle bioenergetics in the Marfan syndrome indicates reduced metabolic efficiency. J Cardiovasc Magn Reson. 2007;9:709-717.

72. Yetman AT, McCrindle BW. The prevalence and clinical impact of obesity in adults with Marfan syndrome. Can J Cardiol. 2010;26:e137-9.

73. Speed TJ, Mathur VA, Hand M, et al. Characterization of pain, disability, and psychological burden in Marfan syndrome. Am J Med Genet A. 2016;173A:315-323

74. Velvin G, Bathen T, Rand-Hendriksen S, Geirdal AØ. Systematic review of chronic pain in persons with Marfan syndrome. Clin Genet. 2016:89:647-658.

75. Fusar-Poli P, Llersy C, Stramesi F, Callegeri A, Arbustini E, Politi P. Determinants of quality of life in Marfan syndrome. Psychosomatics. 2008;49:243-248. 